\title{
Retention of hexanucleotide repeat- containing intron in C9orf72 mRNA: implications for the pathogenesis of ALS/FTD
}

Michael Niblock', Bradley N. Smith¹, Youn-Bok Lee ${ }^{1}$, Valentina Sardone ${ }^{1}$, Simon Topp ${ }^{1}$, Claire Troakes ${ }^{1,2}$, Safa Al-Sarraj ${ }^{2}$, Claire S. Leblond ${ }^{3}$, Patrick A. Dion ${ }^{3}$, Guy A. Rouleau ${ }^{3}$, Christopher E. Shaw ${ }^{1}$ and Jean-Marc Gallo ${ }^{1 *}$

\begin{abstract}
Introduction: The most common forms of amyotrophic lateral sclerosis and frontotemporal dementia are caused by a large GGGGCC repeat expansion in the first intron of the C9orf72 gene. The repeat-containing intron should be degraded after being spliced out, however GGGGCC repeat-containing RNA species either accumulate in nuclear foci or are exported to the cytoplasm where they are translated into potentially toxic dipeptide repeat proteins by repeat-associated non-AUG-initiated (RAN) translation.

Results: In order to determine the mechanisms of repeat-containing intron misprocessing, we have analyzed C9orf72 transcripts in lymphoblasts from C9orf72 expansion carriers $(n=15)$ and control individuals $(n=15)$. We have identified polyadenylated C9orf72 RNA species retaining the repeat-containing intron and in which downstream exons are spliced correctly resulting in a C9orf72 mRNA with an enlarged 5'-UTR containing the GGGGCC repeats. Intron-retaining transcripts are produced from both wild-type and mutant alleles. Intron-retaining C9orf72 transcripts were also detected in brain with a 2.7 fold increase measured in the frontal cortex from heterozygous expansion carriers $(n=11)$ compared to controls $(n=10)$. The level of intron-retaining transcripts was increased 5.9 fold in a case homozygous for the expansion. We also show that a large proportion of intron 1retaining C9orf72 transcripts accumulate in the nucleus.

Conclusions: Retention of the repeat-containing intron in mature C9orf72 mRNA can potentially explain nuclear foci formation as well as nuclear export of GGGGCC repeat RNA and suggests that the misprocessing of C9orf72 transcripts initiates the pathogenic process caused by C9orf72 hexanucleotide repeat expansions as well as provides the basis for novel therapeutic strategies.
\end{abstract}

Keywords: C9orf72, Amyotrophic lateral sclerosis, Frontotemporal dementia, RNA, Repeats, Splicing

\section{Introduction}

Amyotrophic lateral sclerosis (ALS), a devastating degenerative disease of motor neurons and frontotemporal dementia (FTD), the second most common form of presenile dementia after Alzheimer's disease, show considerable overlap clinically and genetically. Pathologically, ALS and FTD patients display abundant cytoplasmic

\footnotetext{
* Correspondence: jean-marc.gallo@kcl.ac.uk

${ }^{1}$ Department of Basic and Clinical Neuroscience, Maurice Wohl Clinical Neuroscience Institute, Institute of Psychiatry, Psychology and Neuroscience, King's College London, 125 Coldharbour Lane, London SE5 9NU, UK Full list of author information is available at the end of the article
}

inclusions of the DNA and RNA-binding protein, TDP43 , suggesting that both neurodegenerative conditions are likely to represent two ends of a single pathological continuum. The most common forms of familial and sporadic ALS and FTD, referred to as C9ALS/FTD, are caused by a non-coding GGGGCC $\left(\mathrm{G}_{4} \mathrm{C}_{2}\right)$ hexanucleotide repeat expansion in the C9orf72 gene on chromosome $9 \mathrm{p} 21[1,2]$. The number of repeats ranges from 2 to 23 in the normal population but is increased to more than 700-1600 repeats in affected individuals. The proportion of sporadic cases with a $\mathrm{G}_{4} \mathrm{C}_{2}$ repeat expansion 
depends on geographical origin. For example, in the United Kingdom, the $\mathrm{G}_{4} \mathrm{C}_{2}$ repeat expansion accounts for $20-50 \%$ of familial or $5-10 \%$ of sporadic cases of ALS $[3,4]$. The $\mathrm{G}_{4} \mathrm{C}_{2}$ repeat expansion is located either in intron 1, between two 5'-untranslated region (5'-UTR) exons, or in the promoter region according to whether an upstream or a downstream transcription start site is used.

In addition to possible haploinsufficiency, two, not mutually exclusive, mechanisms have been proposed to explain the pathogenesis of c9ALS/FTD: RNA transdominant toxic effects and repeat-associated non-AUGinitiated (RAN) translation of $\mathrm{G}_{4} \mathrm{C}_{2}$ RNA repeats into potentially toxic dipeptide repeat proteins (DPRs) [5-7]. The $G_{4} C_{2}$ repeats are transcribed from both sense and antisense strands of the C9orf72 gene [5, 8-11]. Sense and antisense $C 9$ orf 72 repeat RNA can form nuclear foci that have been detected in brain and spinal cord tissue from affected individuals [1, 5, 9-13] and in neurons differentiated from induced pluripotent stem cells (iPSCs) established from c9ALS/FTD patients [12, 14, 15]. Sequestration of specific RNA-binding proteins by hexanucleotide RNA repeats could impair their RNA processing activity and contribute to pathogenesis. Proteins binding to $\mathrm{G}_{4} \mathrm{C}_{2}$ repeats and co-localizing with foci identified to date include hnRNP A3 [16], Pur $\alpha$ [17], ADARB2 [12], hnRNP $H[13,18]$ and nucleolin [19]. Sense and antisense RNA repeats can also be exported to the cytoplasm where they are each translated into the three possible reading frames by RAN translation resulting in five DPRs that have been detected in the brain of c9ALS/FTD patients [5-9]. Arginine-rich DPRs (GR, PR) causes neurodegeneration in Drosophila [20] or are toxic in transfected cells [21-24].

Impairment of nucleocytoplasmic transport appears to be a key mediator of C9orf72-linked pathogenesis. For instance, components of the nuclear pore complex have been identified as modifiers of pathogenesis in several model systems [25-28] and the C9orf72 protein itself binds to components of the nuclear pore complex and its short isoform has been shown to relocalize from the nuclear membrane to the plasma membrane in neurons from expansion carriers [29].

C9orf72 intron 1 , where $\mathrm{G}_{4} \mathrm{C}_{2}$ repeats are located, should be degraded after being spliced out during the processing of pre-mRNA into mature mRNA. However, in c9ALS/FTD, expanded $\mathrm{G}_{4} \mathrm{C}_{2}$ repeats fail to be degraded and form nuclear foci or are RAN translated into DPRs. The mechanisms of defective nuclear degradation as well as of nuclear export of C9orf $72 \mathrm{G}_{4} \mathrm{C}_{2}$ repeat sequences are presently unknown but are central to the pathogenesis of c9ALS/FTD. Interestingly, treatment with antisense oligonucleotides (ASOs) targeting C9orf72 exons downstream of intron 1, up to exon
11, promotes degradation of expanded C9orf72 transcripts [11, 12, 15]. As splicing occurs mainly cotranscriptionally this suggests that intron 1 is still present in nascent transcripts when downstream exons have been spliced. This prompted us to analyze the fate of C9orf72 intron 1 in cells derived from expansion carriers.

Here we identify polyadenylated C9orf72 RNA species retaining the repeat-containing intron and in which downstream exons are spliced correctly resulting in a C9orf72 mRNA with an enlarged 5'-UTR containing the $\mathrm{G}_{4} \mathrm{C}_{2}$ repeats. Generation of intron 1-retaining RNA species potentially explains a number of pathological features of c9ALS/FTD and opens the way to novel therapeutic strategies.

\section{Materials and methods Lymphoblasts}

Lymphoblasts from C9orf72 expansion carriers $(n=15)$ were generated using a standard protocol where the Epstein-Barr virus is used to immortalize B-lymphocytes or were obtained from the UK MND DNA Bank. Control lymphoblasts from individuals free from neurological disease $(n=15)$ were generated as above or were from the European Collection of Animal Cell Cultures (ECACC). Cells have been genotyped for the C9orf72 $\mathrm{G}_{4} \mathrm{C}_{2}$ hexanucleotide repeat expansion by repeat-primed PCR $[1,2]$. Lymphoblasts were grown in RPMI supplemented with $15 \%(\mathrm{v} / \mathrm{v}) \mathrm{FBS}, 100 \mathrm{UI} / \mathrm{ml}$ penicillin and $100 \mu \mathrm{g} / \mathrm{ml}$ streptomycin.

\section{Brain tissue}

Frozen brain tissue from cases with frontotemporal lobar degeneration (FTLD) or frontotemporal lobar degeneration with motor neuron disease (FTLD-MND) $(n=11)$ and control cases from individuals free from neurological disease $(n=10)$ were obtained from the MRC London Neurodegenerative Diseases Brain Bank (Institute of Psychiatry, Psychology and Neuroscience, King's College London, UK), and were collected in accordance with local and national research ethics guidelines (Additional file 1: Table S1). The C9orf72 status of the cases used was confirmed by repeat-primed PCR.

\section{RNA isolation}

Total RNA was extracted from whole lymphoblasts or from cytoplasmic and nuclear fractions (see below) using TRIzol (Life Technologies). Frozen brain tissue samples were homogenized in matrix lysing-D tubes (MP Biomedicals) in conjunction with the Fastprep sample preparation system and total RNA was extracted using the RNeasy lipid tissue kit (Qiagen). RNA from an FTLD case homozygous for the C9orf $72 \mathrm{G}_{4} \mathrm{C}_{2}$ repeat expansion [30] was obtained from Dr Adrian Isaacs and Dr Pietro 
Fratta (UCL Institute of Neurology, London). Any residual contaminating genomic DNA was eliminated by treatment with Turbo DNase (Ambion). Poly $(\mathrm{A})^{+}$RNA was selected from total RNA using oligo(dT) conjugated to magnetic beads (Dynabeads ${ }^{\circ}$ Oligo $(\mathrm{dT})_{25}$, Ambion) according to the manufacturer's protocol. RNA concentration was determined with a Nanodrop spectrophotometer (Thermo Scientific). RNA integrity number (RIN) was measured with an Agilent RNA 6000 analyzer (lymphoblasts, controls: 6.0-7.6, expansion carriers: 5.97.5; brain, controls: 3.9-6.5, expansion carriers: 3.8-5.7).

\section{Nuclear and cytoplasmic fractionation}

Nuclear and cytoplasmic fractionation was carried out using a modification of earlier protocols [31-33]. Lymphoblasts were centrifuged at $800 \mathrm{rpm}$ for $5 \mathrm{~min}$ and lysed

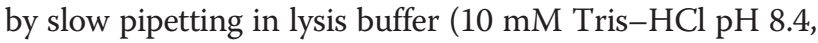
$140 \mathrm{mM} \mathrm{NaCl}, 1.5 \mathrm{mM} \mathrm{MgCl} 2,0.5 \%$ Nonidet P-40, $1 \mathrm{mM}$ dithiothreitol and $100 \mathrm{U} / \mathrm{ml}$ RNasin). The suspension was centrifuged at $1000 \mathrm{~g}$ for $3 \mathrm{~min}$ at $4{ }^{\circ} \mathrm{C}$ and the supernatant recovered as the cytoplasmic fraction. $\mathrm{Nu}-$ clear pellets were resuspended in lysis buffer with $3.3 \%$ (w/v) sodium deoxycholate and $6.6 \%(\mathrm{v} / \mathrm{v})$ Tween 40 . The samples were vortexed and incubated on ice for $5 \mathrm{~min}$. Nuclei were re-pelleted by centrifugation at $1000 \mathrm{~g}$ for $3 \mathrm{~min}$ at $4{ }^{\circ} \mathrm{C}$. Pooled supernatants were centrifuged at $1000 \mathrm{~g}$ for $5 \mathrm{~min}$ at $4{ }^{\circ} \mathrm{C}$ and transferred to fresh tubes. Nuclei were lysed in thiocyanate buffer (4 M guanidinium thiocyanate, $20 \mathrm{mM}$ sodium acetate, $0.1 \mathrm{mM}$ dithiothreitol, $0.5 \%(\mathrm{w} / \mathrm{v})$ sarkosyl). RNA was extracted from cytoplasmic and nuclear fractions using TRIzol, as above. Final RNA volumes from each fraction were adjusted to represent cell-equivalent concentrations [34].

\section{RT-PCR}

RNA was reverse transcribed using the TaqMan RT kit or the Superscript III kit (Life Technologies) with oligo(dT) or random hexamers according the manufacturer's protocols. Reverse-transcribed RNA was amplified by PCR using GoTaq polymerase (Promega) using primers and conditions detailed in Additional file 1: Table S2. For PCR across the $\mathrm{G}_{4} \mathrm{C}_{2}$ repeats, reactions were supplemented with betaine (Sigma), DMSO and 7deaza GTP (New England Biosystems) [1]. No RT controls were used to confirm absence of DNA contamination. RT-PCR products were separated in $1.5 \%(\mathrm{w} / \mathrm{v})$ agarose gels and stained with ethidium bromide. The amount of PCR product was estimated by densitometric analysis of the gels using the VisionWorks ${ }^{\circ} \mathrm{LS}$ analysis software (UVP).

\section{qRT-PCR}

For real-time quantitative PCR, reactions contained $5 \mu \mathrm{l}$ SYBR Green (Roche Diagnostics), $1.25 \mu \mathrm{M}$ of each primer and 10 ng of cDNA. Primers (Additional file 1: Table S2) were designed using Primer-BLAST. Each primer pair produced a single PCR product and its identity was confirmed by sequencing. To produce standard curves for absolute quantification, PCR products were ligated into the pGEM-T easy vector and transformed into JM109 E. coli. Concentrations of plasmid DNA were determined using a Nanodrop spectrophotometer (Thermo Scientific) and copy numbers calculated. Serial dilutions were made to produce standard curves ranging from $10^{1}$ to $10^{7}$ molecules (Additional file 1: Figure S5). qPCR was performed in 384-well plates on an Applied Biosystems 7900HT Fast Real-Time PCR System using the conditions detailed in Additional file 1: Table S2. Samples were run in duplicate and the average cycle threshold $(\mathrm{Ct})$ was calculated for target and standards. These values were used to calculate the number of RNA molecules in each sample. Statistical analysis was carried out using the Mann-Whitney $U$ test using the SPSS software.

\section{Sequencing}

PCR products were excised from the gels and extracted using the Qiaquick gel extraction kit (Qiagen) and cloned into the pGEM-T Easy vector (Promega) using the TA cloning system. Sequencing of both strands was performed commercially using SP6 and T7 primers (MWG Eurofins). For allele analysis, a 451 bp fragment spanning the intron 1-exon 2 boundary and the rs10757668 SNP was amplified by PCR using standard procedures. Amplicons were directly sequenced with the same primers using Big-Dye Terminator v1.1 and products run on an ABI3130 Genetic Analyzer (Applied Biosystems).

\section{Results}

Intron 1 retention in polyadenylated C9orf72 transcripts in lymphoblasts from $\mathrm{G}_{4} \mathrm{C}_{2}$ expansion carriers

Defective splicing of $C 9$ orf72 intron 1 could result in its retention in an otherwise mature mRNA. To determine whether polyadenylated RNA contained sequences derived from $C 9$ orf 72 intron 1, we analyzed poly(A) ${ }^{+}$RNA from cultured lymphoblasts established from heterozygous $C 9$ orf $72 \mathrm{G}_{4} \mathrm{C}_{2}$ repeat expansion carriers and control individuals. Lymphoblasts derived from C9orf $72 \mathrm{G}_{4} \mathrm{C}_{2}$ repeat expansion carriers display nuclear foci [11], thus, abnormal processing of intron 1 occurring in neurons is likely to be recapitulated in lymphoblasts.

Poly(A) ${ }^{+}$RNA was purified from lymphoblasts and reverse transcribed using an oligo(dT) primer. The lack of contaminating genomic DNA was demonstrated by PCR using primers spanning a short intron in the HOXB4 gene [35] (Additional file 1: Figure S1a). The large size and high GC content of intron 1 prevents its direct 
amplification by PCR. To determine whether intron 1 sequences were present in polyadenylated C9orf72 RNA, cDNA was analyzed by PCR using primers spanning the exon 1a-intron 1 and intron 1-exon 2 boundaries. PCR with a forward primer specific for exon $1 \mathrm{a}$ and a reverse primer annealing to intron 1,5 'of the $\mathrm{G}_{4} \mathrm{C}_{2}$ repeats, generated a $278 \mathrm{bp}$ product from both control and expansion carrier cells (Fig. 1a, left). PCR with a forward primer annealing to intron 1 , 3'of the $\mathrm{G}_{4} \mathrm{C}_{2}$ repeats, and a reverse primer specific for exon 5 generated a $1156 \mathrm{bp}$ product from both control and expansion carrier cells
(Fig. 1a, right). Such products were not observed in control reactions with no reverse transcriptase, ruling out the possibility that they originated from residual contaminating genomic DNA (Additional file 1: Figure S2). Oligo(dT) was used as a primer for reverse transcription in order to add a further level of specificity; the same results were obtained when reverse transcription was primed using random hexamers (Additional file 1: Figure S3). Thus, mature polyadenylated RNA species derived from $C 9$ orf 72 and containing intron 1 sequences are produced in lymphoblasts from expansion carriers and

a

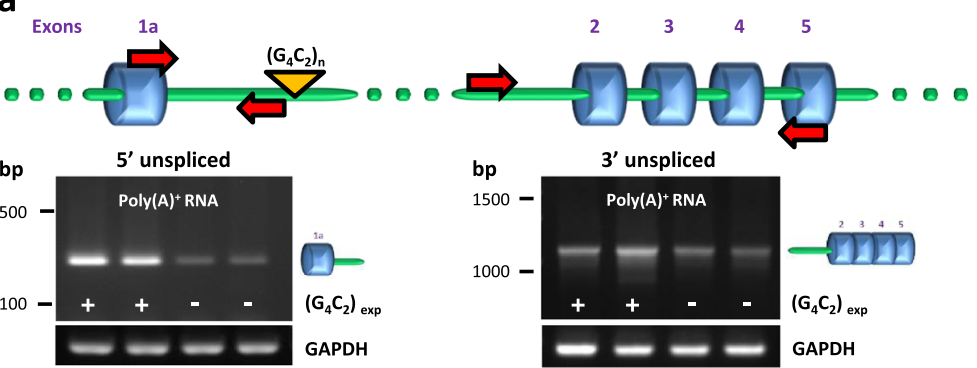

b
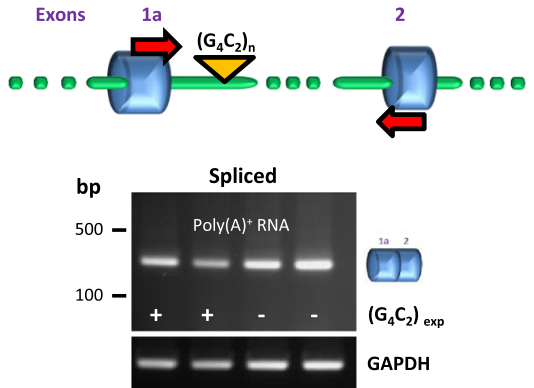

C
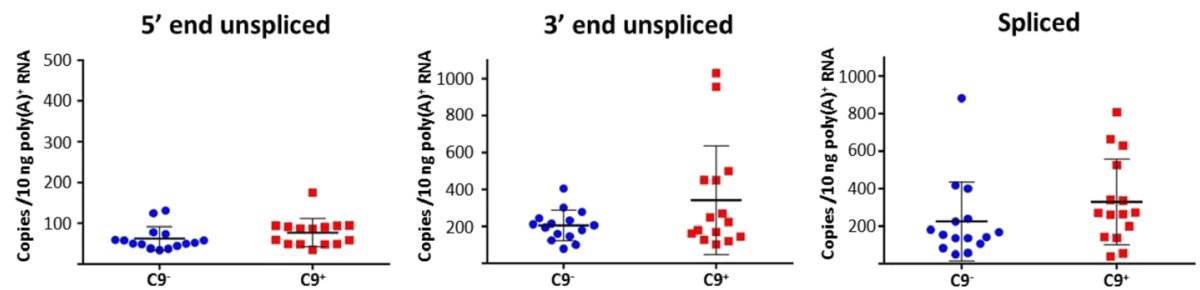

d

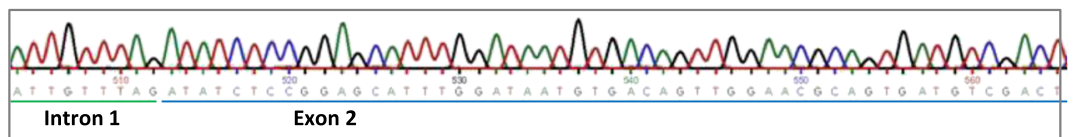

Fig. 1 Intron 1 retention in C9orf72 transcripts in lymphoblasts. C9orf72 transcripts were analyzed in lymphoblasts from C9orf72 $\mathrm{G}_{4} \mathrm{C}_{2}$ expansion carriers and control individuals. a RT-PCR analysis of poly $(A)^{+}$RNA using primers spanning the $5^{\prime}$ splice site (left) or the $3^{\prime}$ splice site (right) of intron 1 demonstrating retention of intron 1in polyadenylated RNA. The position of the primers is indicated on the diagram above the gels. GAPDH was used as a loading control. b Correctly spliced transcripts detected in controls and expansion carrier cells using primers in exons 1a and 2. c Quantitative analysis of intron retention by real-time PCR. Levels of C9orf72 transcripts spliced or unspliced at the 5' and 3' end of intron 1 were determined by real-time qRT-PCR. Data are shown as means \pm SEM. Each data point represents an individual case, $n=15$ (C9); 15 (C9+). No significant differences were observed between the $C 9^{-}$and $C 9^{+}$groups. $\mathbf{d}$ Sequencing of the $3^{\prime}$ PCR product demonstrates an exact intron 1-exon 2 boundary. $\mathrm{C9}^{-}$, controls; $\mathrm{C9}^{+}$, expansion carriers 
control individuals. In addition to transcripts retaining intron 1 C9orf 72 transcripts in which intron 1 has been correctly spliced out were also detected in both control cells and cells from expansion carriers (Fig. 1b).

Intron 1 retention was quantified by measuring the levels of C9orf72 transcripts unspliced at the 5' end or at the 3' end by real-time quantitative RT-PCR (qRT-PCR) in lymphoblasts from expansion carriers $(n=15)$ and controls $(n=15)$. No significant differences were detected between the two groups (Fig. 1c). Comparison with normally spliced transcripts shows that about $25 \%$ of $C 9$ orf 72 transcripts retain intron 1 . This value is comparable with what has been measured for several transcripts in granulocytes [36].

Sequencing confirmed that the 5' PCR product contained an exact exon 1a-intron 1 boundary and that the 3 ' product contained an exact intron 1-exon 2 boundary as well as exon 2-3, 3-4 and 4-5 junctions (Fig. 1d and Additional file 1: Figure S4). The latter product corresponds to a transcript retaining intron 1 and in which the other introns have been spliced out correctly, at least up to intron 4. HOXB4 analysis and RT negative controls effectively rule out the possibility that the products detected had originated from genomic DNA (Additional file 1: Figures S1a and S2). Furthermore, the 3' end product, that contains exact exon-exon junctions can only have originated from a reverse transcribed RNA.

Intron 1-retaining transcripts contain the repeat sequence and are produced from both wild-type and mutant alleles Detection of exact exon 1a-intron 1 and intron 1-exon 2 boundaries in cDNA suggests that the template RNA overlaps the $\mathrm{G}_{4} \mathrm{C}_{2}$ repeat sequence. To confirm that polyadenylated C9orf72 RNA contained the repeat sequence, we performed a PCR on cDNA using a pair of primers spanning the repeat region. PCR across repeats will only amplify the product from the wild-type allele in expansion carrier cells, as the repeat region from the expanded allele cannot be amplified due to its size and high GC content. Using this method, two products of slightly different sizes were detected in cells from normal individuals and only one product was generated from expansion carrier cells (Fig. 2a). Such products were not observed in control reactions in which reverse transcriptase was omitted, ruling out the possibility that they originated from residual contaminating intronic genomic DNA (not shown). Therefore the repeat region of the two alleles in control cells and of the wild-type allele in expansion carrier cells is present in polyadenylated C9orf72 transcripts.

To determine whether intron 1-retaining transcripts could be produced from both the wild-type and the mutant allele, we took advantage of the G>A singlenucleotide polymorphism (SNP) rs10757668 in C9orf72 exon 2 [1]. We first selected two expansion carrier cases heterozygous for rs10757668 from cases that had been genotyped previously [3]. An intron 1-exon 2 fragment overlapping rs10757668 was amplified and sequenced from genomic DNA, as confirmation of heterozygosity for rs10757668, and from cDNA reversed transcribed from $\operatorname{poly}(\mathrm{A})^{+}$RNA. Sequence analysis revealed that intron 1-retaining transcripts contained both the $G$ and A alleles showing that intron 1 retention occurred in RNA transcribed from the wild-type as well as the expanded allele (Fig. 2b).

\section{Intron 1-retaining C9orf72 transcripts accumulate in the nucleus}

A feature of intron-retaining transcripts is their failure to be exported from the nucleus [36, 37]. We therefore compared the partitioning of C9orf72 transcripts between the nucleus and the cytoplasm in lymphoblasts from $\mathrm{G}_{4} \mathrm{C}_{2}$ repeat expansion carriers and control cases. Nuclear and cytoplasmic fractions were prepared and poly $(\mathrm{A})^{+}$RNA was extracted from each fraction and reverse transcribed. $H O X B 4$ analysis confirmed the lack of contaminating genomic DNA in both fractions (Additional file 1: Figure S1b). The purity of the nuclear and cytoplasmic fractions was further assessed by analyzing the polyadenylated nuclear long non-coding RNA, NEAT1 [38]. NEAT1 was only found in the nuclear fraction, demonstrating that the cytoplasmic fraction was free of nuclear contamination (Fig. 3a). Poly(A) ${ }^{+}$C9orf72 transcripts from both fractions were analyzed for defective splicing of intron 1 at the 5' and 3' ends or splicing out of intron 1 (Fig. 3a). Quantification of nuclear and cytoplasmic contents showed that approximately $85 \%$ of C9orf72 transcripts retaining intron 1 were found in the nuclear fraction whereas correctly spliced transcripts were predominantly cytoplasmic (Fig. 3b). RNA unspliced at the 3' end and RNA unspliced at the 5' end were also detected, albeit at low levels, in the cytoplasmic fraction (Fig. 3b).

\section{Intron 1-retaining C9orf72 transcripts in brain}

We next determined whether intron 1-retaining C9orf72 transcripts were present in brain tissue from $C 9$ orf 72 hexanucleotide expansion carriers. RNA from the frontal cortex from heterozygous expansion carriers and from control cases was extracted and analyzed for intron 1 retention. C9orf72 transcripts unspliced at the 5' or 3' end of intron 1 were detected in both control and expansion carriers (Fig. 4a). We also analyzed intron 1 retention in the frontal cortex from an FTLD case homozygous for the C9orf72 $\mathrm{G}_{4} \mathrm{C}_{2}$ repeat expansion in which results would not confounded by the presence of transcripts from the wild-type allele [30]. The levels of unspliced C9orf72 transcripts were markedly higher in the 

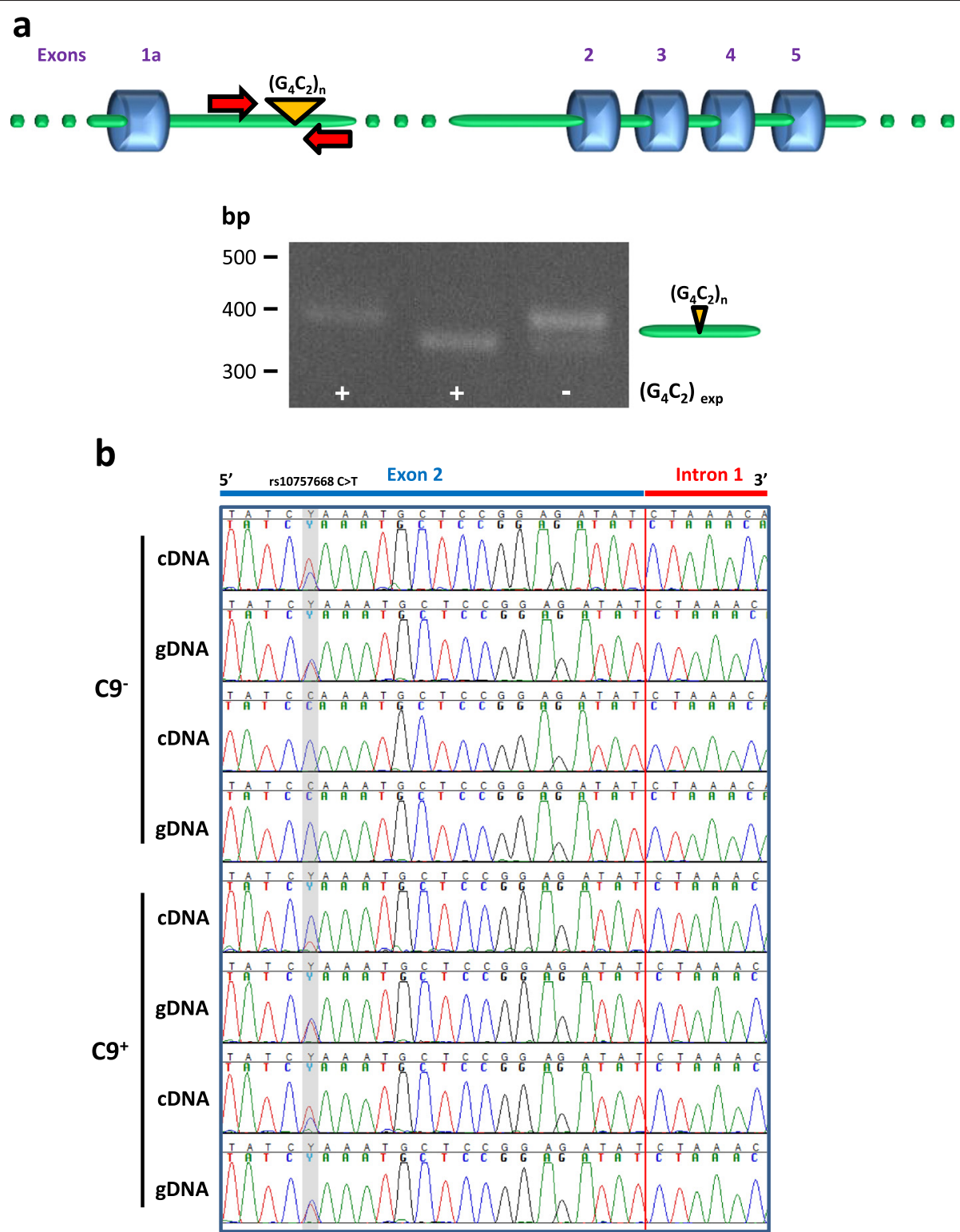

Fig. 2 a Intron 1-retaining transcripts contain the $G_{4} C_{2}$ repeat sequence. PCR using primers spanning the $G_{4} C_{2}$ repeat region was performed on CDNA reversed transcribed from polyadenylated C9orf72 RNA from control and expansion carrier lymphoblasts. The two products of slightly different sizes detected in cells from normal individuals correspond to the two alleles; the single product generated from expansion carrier cells corresponds to the wild-type allele. $\mathbf{b}$ Intron 1-retaining transcripts are produced from both wild-type and mutant alleles. Sequence traces of C9orf72 overlapping the intron 1-exon 2 boundary and the rs10757668 SNP in cDNA (top panels) and genomic DNA (gDNA, bottom panels) prepared from lymphoblasts from two control individuals and two expansion carriers heterozygous for rs 10757668 . One of the control cases (top trace) is heterozygous for rs 10757668 . The sequence of the reverse strand is shown; the position of rs 10757668 is indicated by the grey box. Intron 1-retaining transcripts contain both the $\mathrm{C}$ and $\mathrm{T}$ alleles showing that intron 1 retention occurs in RNA transcribed from the wild-type as well as the expanded allele. $\mathrm{C9}^{-}$, controls; $\mathrm{C9}^{+}$, expansion carriers

homozygous case than in heterozygous cases (Fig. 4a). The levels of C9orf72 transcripts unspliced at the 5' end or at the 3' end were measured by qRT-PCR as for lymphoblasts (Fig. 4b). The level of C9orf72 transcripts unspliced at the 5' end in the frontal cortex from heterozygous expansion carriers $(n=11)$ showed a statistically significant 2.7 fold increase compared to control cases $(n=10)$ (Fig. 4b). The level of transcripts unspliced at the 5 ' was increased by 5.9 fold in the homozygous case analyzed.

\section{Discussion}

Here, we have identified previously unrecognized RNA species derived from polyadenylated C9orf72 RNA that 


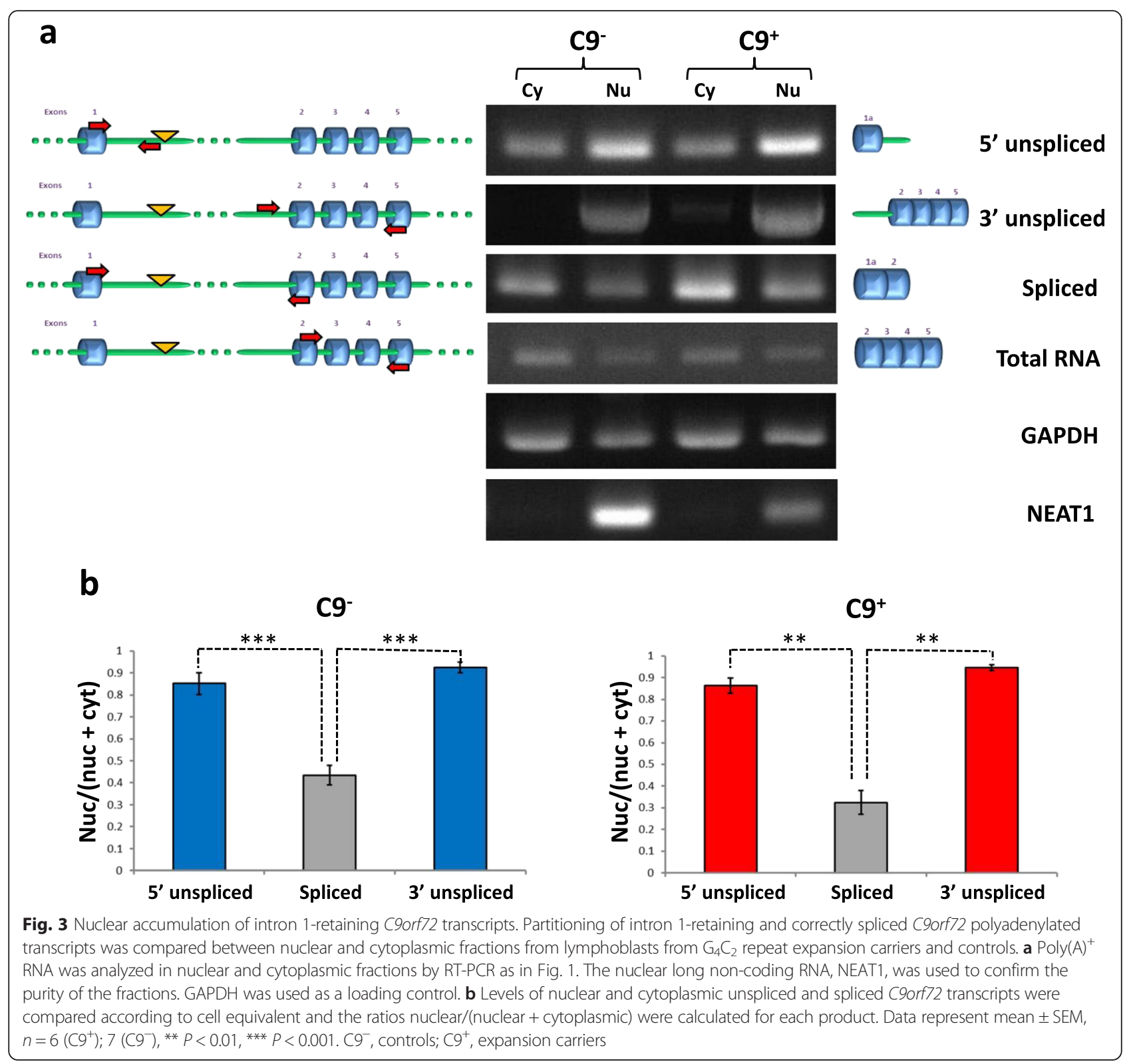

are unspliced at the $5^{\prime}$ and $3^{\prime}$ end of intron 1 and in which downstream exons are spliced correctly. As intron 1 is located between two 5 '-UTR exons, its retention results in a C9orf72 mRNA with an enlarged 5'-UTR. Interestingly, higher levels of intron 1-retaining C9orf72 RNA species were detected in the frontal cortex of expansion carriers compared to control individuals. Two recent papers also reported increased levels of sense and antisense C9orf72 RNA containing intron 1 in the frontal cortex from expansion carriers $[6,39]$. However, these reports did not address the molecular nature of the RNA species containing intron 1 that accumulate in disease. Intron-containing RNA can have various origins; for example, it could represent incompletely processed
pre-mRNAs stalled during the splicing process, splicedefective lariat intermediates or aborted transcripts [19, 40]. Here we demonstrate that intron 1-containing C9orf72 RNA that accumulates in the brain from expansion carriers is, at least in part, a fully processed mRNA retaining intron 1 within an enlarged 5'-UTR. This has important mechanistic implications for the pathogenic process, as discussed below. No significant difference was found in the levels of transcripts unspliced at the 3' end of intron 1 in brain. This could be explained by the fact that RNA unspliced at the 5' end of intron 1 originates from transcription variants that contains the hexanucleotide repeat sequence, whereas RNA unspliced at the 3' end of intron 1 can, in addition, originate from 

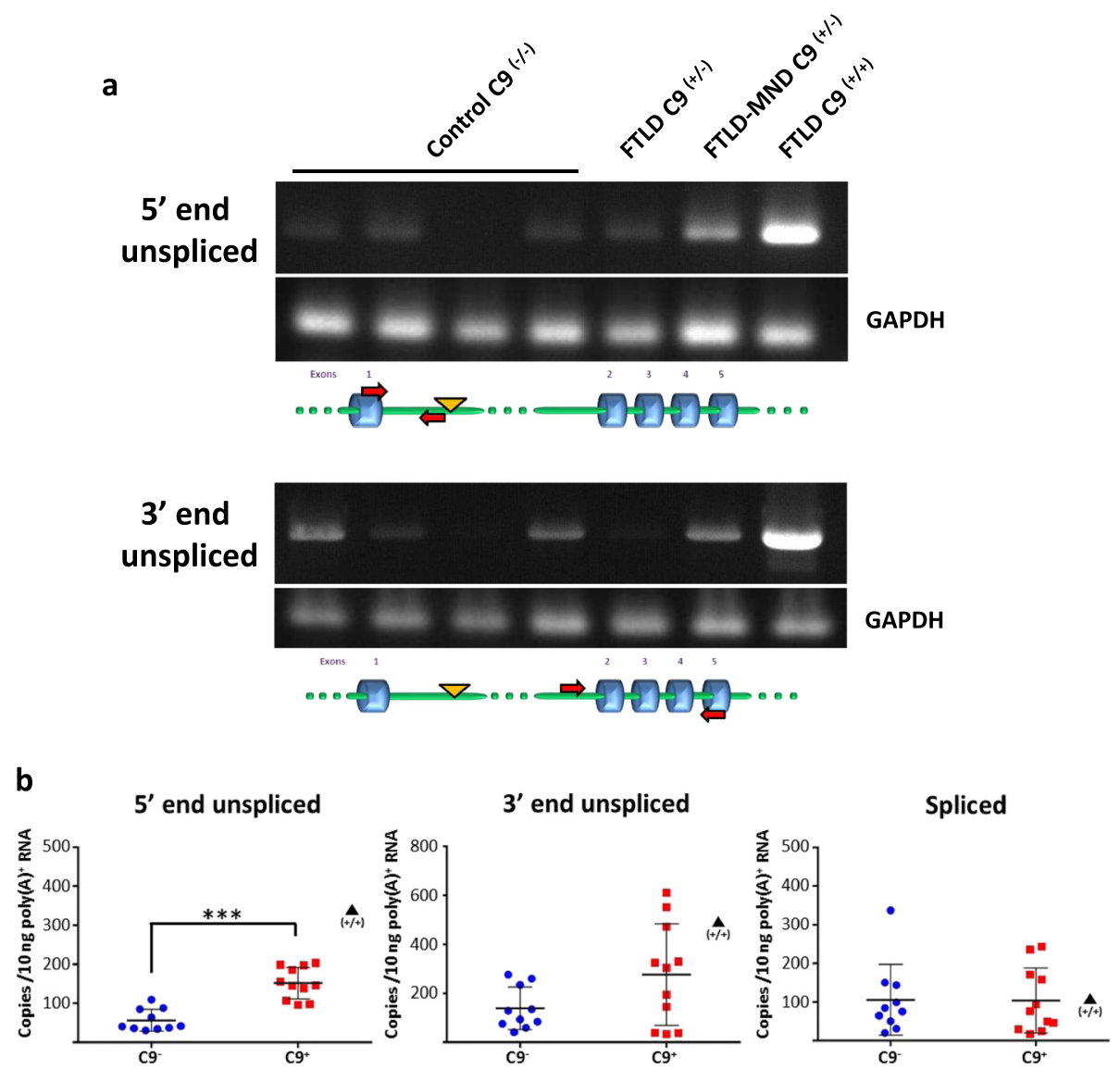

Fig. 4 Intron 1 retention in C9orf72 transcripts in the brain of C9orf72 expansion carriers. C9orf72 transcripts were analyzed in the frontal cortex from frontotemporal lobar degeneration (FTLD) or frontotemporal lobar degeneration with motor neuron disease (FTLD-MND) cases with confirmed C9orf72 hexanucleotide expansions and from control individuals. a RNA was analyzed by RT-PCR as in Fig. 1. GAPDH was used as a loading control. The level of C9orf72 transcripts unspliced at the $5^{\prime}$ and $3^{\prime}$ ends in an FTLD case homozygous for the $\mathrm{C}^{\circ}$ orf72 $\mathrm{G}_{4} \mathrm{C}_{2}$ repeat expansion was markedly higher than in heterozygous cases $\left(C 9^{(++)}\right.$, far right lane). b Quantitative analysis of intron retention by real-time PCR. Levels of C9orf72 transcripts spliced or unspliced at the $5^{\prime}$ and $3^{\prime}$ end of intron 1 were determined by real-time qRT-PCR in heterozygous expansion carriers $(n=11)$ and control cases $(n=10)$. Data are shown as means \pm SEM. Each data point represents an individual case, ${ }^{* * * P}<0.001$, Mann-Whitney $U$ test. $\mathrm{CP}^{-}$, controls; $\mathrm{C9}^{+}$, expansion carriers. $\mathbf{\Lambda}(+/+)$ indicates the values for the single homozygous case analyzed

transcription variants not containing the repeat sequence.

Intron 1 retention is consistent with the degradation of expanded repeat-containing transcripts induced by RNase H-sensitive ASOs targeting C9orf72 RNA up to exon 11 [11, 12, 15]. As splicing out of introns occurs, in most cases, early during transcription, intron 1 is likely not to have been spliced out when transcription has reached exon 11 . The presence of the expansion in a mature mRNA in patient tissue is also consistent with a recent report showing that the repeat expansion has to be in the context of an mRNA to cause toxicity in Drosophila [41].

Intron 1-retention was observed in C9orf72 polyadenylated RNA from both the wild-type and the expanded alleles and contains the $\mathrm{G}_{4} \mathrm{C}_{2}$ repeats, and, thus, is part of a normal process. This is not an unusual situation as intron detention (delayed splicing) or retention, either arising from defective splicing or as a regulatory process, is a common occurrence within the mammalian transcriptome [36, 37, 42, 43]. Although retention of intron 1 in C9orf72 transcripts appears to be independent of the presence of an expanded $\mathrm{G}_{4} \mathrm{C} 2$ repeat sequence, this process results in the expanded $\mathrm{G}_{4} \mathrm{C}_{2}$ sequence from the mutant allele being included in the 5'-UTR of a fully processed mRNA. C9orf72 mRNA with an enlarged 5'UTR that includes the $\mathrm{G}_{4} \mathrm{C}_{2}$ repeats, is similar to FMR1 transcripts associated with fragile $\mathrm{X}$-associated tremor ataxia syndrome (FXTAS), caused by moderate $(<200)$ CGG repeat expansions in the 5 -UTR of the FMR1 gene [44].

In addition to transcripts retaining the entire intron 1 our analysis does not rule out the presence of shorter RNA species, for example resulting from the use of 
cryptic polyadenylation sites in intron 1 . Use of cryptic intronic polyadenylation sites has been reported in Huntington's disease, caused by a CAG repeat expansion in exon 1 of the HTT gene, resulting in a truncated, aggregation prone, protein [45].

No difference in the level of intron retention was detected between lymphoblasts from expansion carriers and controls. By contrast C9orf72 transcripts unspliced at the 5 ' end of intron 1 were found to be expressed at higher levels in the frontal cortex of expansion carriers compared to control individuals. This was particularly clear in the brain of a homozygous case albeit material from one case only was available for analysis. As suggested by Mori et al. [6] the increased levels observed could reflect stabilization of expanded intron 1-containing transcripts. Alternatively, long $\mathrm{G}_{4} \mathrm{C}_{2}$ sequences could also reduce splice site usage, hence inhibiting intron 1 splicing. Of note, introns with a high GC content are prone to be retained [36]. Partial retention of expanded CCTG repeat-containing first intron of the CCHC-type zinc finger (CNBP, formerly known as ZNF9) gene has also been reported in myotonic dystrophy type 2 (DM2) [46]. Long $\mathrm{G}_{4} \mathrm{C}_{2}$ repeat tracts might also slow down the rate of transcription of the C9orf 72 gene in expansion carriers, hence promoting intron retention.

We found that a large proportion of intron 1-retaining C9orf72 transcripts accumulated in the nucleus. Nuclear retention of incompletely spliced transcripts has been demonstrated as a regulated process for the control of gene expression or as part of a surveillance pathway (for reviews see [47-49]). Consistent with this notion, intron-containing transcripts resulting from defective splicing in heat-shocked cells are retained in the nucleus [37]. Nuclear accumulation of C9orf 72 mRNA retaining intron 1 could be the consequence of disrupted nucleocytoplasmic transport of mRNAs resulting from C9orf72 repeat expansion toxicity $[26,50]$. However, this is unlikely to be the case as correctly spliced transcripts (e.g. spliced C9orf72) are prevalent in the cytoplasm. Of note, huntingtin mRNA with expanded CAG repeats accumulates in the nucleus [51].

mRNAs that fail to be exported to the cytoplasm are degraded in the nucleus by a surveillance pathway that has been studied in yeast and reported, but yet to be fully characterized, in mammals [52, 53]. This pathway may involve poly(A)-binding proteins (PABPs) and nuclear exosomes [54-56]. Intron 1-retaining C9orf72 mRNA may be targeted to such a pathway that would be unable to destabilize the G-quadruplex structure formed by the $G_{4} C_{2}$ repeat sequence $[19,57-59]$ and the repeat region would be protected from degradation, resulting in its accumulation in foci (Fig. 5). While the above pathway may be the pathway to which intron-retaining C9orf72 mRNA is targeted, it is important to note that RNA can be degraded through different pathways.

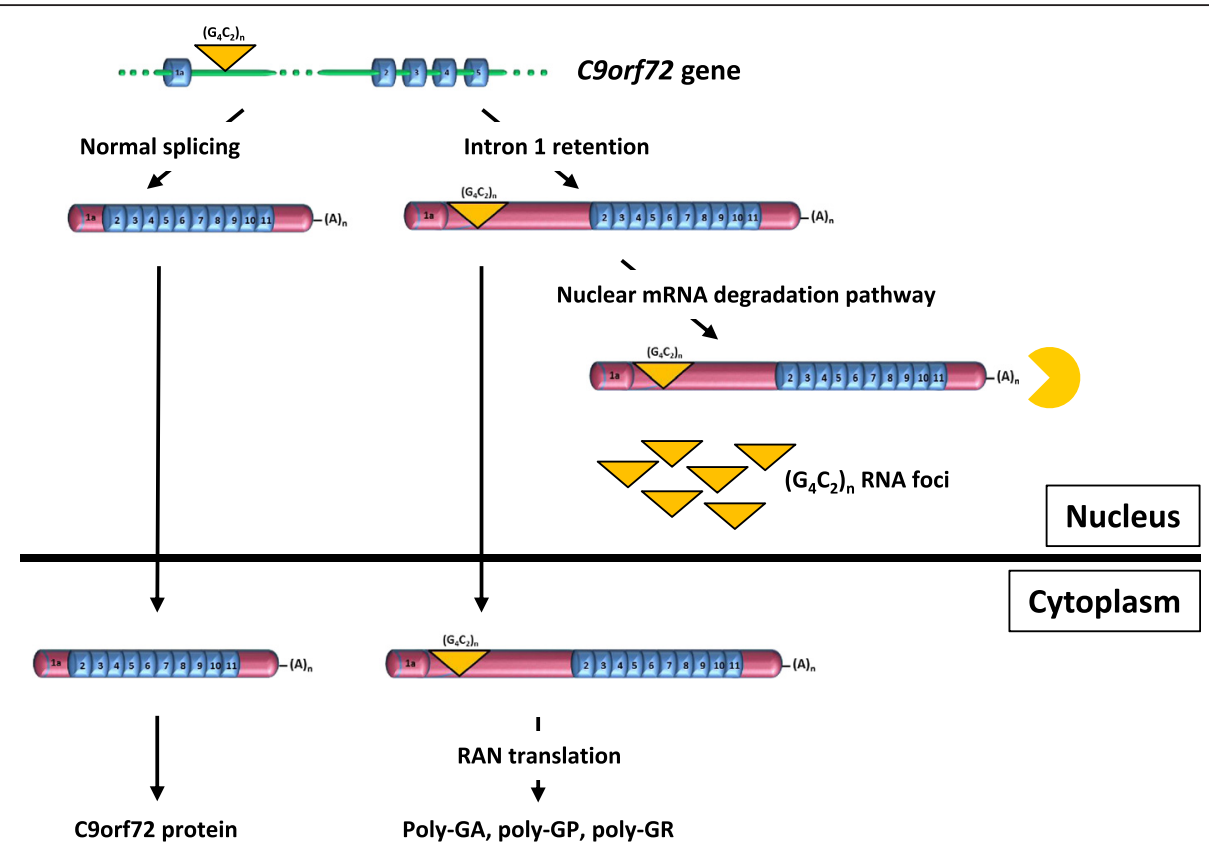

Fig. 5 Model of expanded C9orf72 transcripts processing explaining the main pathological features of C9ALS/FTD. Retention of intron 1 generates a C9orf72 mRNA with an enlarged 5'-UTR containing the $\mathrm{G}_{4} \mathrm{C}_{2}$ repeat sequence. The majority of intron 1-retaining C9orf72 mRNA accumulates in the nucleus where it is targeted to a specific degradation pathway unable to process $G_{4} C_{2}$ RNA repeats that subsequently aggregate into foci. $A$ small proportion of intron 1-retaining C9orf72 mRNA is exported to the cytoplasm for RAN translation into DPRs 
Indeed, as mentioned above, long $\mathrm{G}_{4} \mathrm{C}_{2}$ repeat sequences can be degraded following ASO-mediated RNase $\mathrm{H}$ cleavage of C9orf72 RNA [11, 12, 15].

Although the majority of intron 1-retaining C9orf72 transcripts accumulate in the nucleus, intron retention could also explain export of the expanded $\mathrm{G}_{4} \mathrm{C}_{2}$ repeat RNA to the cytoplasm where it would become template for RAN translation into DPRs. As intron 1-retaining C9orf72 transcripts have the structure of mature mRNAs, an, albeit small, proportion is exported to the cytoplasm through the conventional pathway of nuclear export of mRNA (Fig. 5). However, this does not exclude that other $\mathrm{G}_{4} \mathrm{C}_{2}$ repeat-containing RNA species might be the main template for RAN translation.

Finally, intron retention in the sense transcript might explain transcription of repeats from the reverse strand. $\mathrm{G}_{4} \mathrm{C}_{2}$ expansions in the C9orf72 gene have been shown to promote the formation of RNA-DNA hybrids (R-loops) [19]. Intron retention as well as formation of R-loops could be linked to the slowing down of transcription of the sense transcript by the repeat sequence [60]. R-loops would, in turn, induce antisense transcription [60].

\section{Conclusions}

We have identified C9orf72 mRNA species with an enlarged 5'-UTR that includes the $\mathrm{G}_{4} \mathrm{C}_{2}$ repeat sequence that can explain a number of features of c9ALS/FTD. Interfering with intron 1 processing would offer novel ways of dissecting the cascade of events leading to neuronal dysfunction and death and represents an innovative and promising therapeutic strategy for c9ALS/FTD.

\section{Additional file}

Additional file 1: Supplementary material. (PDF $456 \mathrm{~kb}$ )

\section{Competing interests}

The authors declare that they have no competing interests.

\section{Authors' contributions}

JMG, MN and CES conceived the study and experimental design, MN carried out RNA analyses, BNS, ST and YBL carried out molecular genetic studies. CT and SAS selected and prepared brain tissue. VS, CSL, PAD and GAR provided lymphoblast cell lines. JMG and MN wrote the manuscript. All authors read and approved the final manuscript.

\section{Acknowledgments}

We thank Dr Adrian Isaacs and Dr Pietro Fratta (UCL Institute of Neurology, London) for material from the homozygous FTLD case used. We thank Rebecca Jones for her help with statistical analyses and Dr Frank Hirth for helpful discussions.

\section{Funding}

This work was supported by the Wellcome Trust, the Medical Research Council, the Motor Neurone Disease Association, Alzheimer's Research UK and the Psychiatry Research Trust. Tissue was provided by the London Neurodegenerative Diseases Brain Bank, which receives funding from the Medical Research Council and from the Alzheimer's Society and Alzheimer's Research UK (through the Brains for Dementia Research project).

\section{Author details}

${ }^{1}$ Department of Basic and Clinical Neuroscience, Maurice Wohl Clinical Neuroscience Institute, Institute of Psychiatry, Psychology and Neuroscience, King's College London, 125 Coldharbour Lane, London SE5 9NU, UK.

${ }^{2}$ London Neurodegenerative Disease Brain Bank, Institute of Psychiatry, Psychology and Neuroscience, King's College London, London SE5 8AF, UK. ${ }^{3}$ Montreal Neurological Institute and Hospital, McGill University, Ludmer Building, 1033 Pine Avenue West, Montreal, QC H3A 1A1, Canada.

Received: 15 February 2016 Accepted: 15 February 2016

Published online: 25 February 2016

\section{References}

1. Dejesus-Hernandez M, Mackenzie IR, Boeve BF, Boxer AL, Baker M, Rutherford NJ, et al. Expanded GGGGCC hexanucleotide repeat in noncoding region of C9ORF72 causes chromosome 9p-linked FTD and ALS. Neuron. 2011;72:245-56. doi:10.1016/j.neuron.2011.09.011.

2. Renton AE, Majounie E, Waite A, Simon-Sanchez J, Rollinson S, Gibbs JR, et al. A hexanucleotide repeat expansion in C9ORF72 is the cause of chromosome 9p21-linked ALS-FTD. Neuron. 2011;72:257-68. doi:10.1016/j. neuron.2011.09.010.

3. Smith BN, Newhouse S, Shatunov A, Vance C, Topp S, Johnson L, et al. The C9ORF72 expansion mutation is a common cause of ALS+/-FTD in Europe and has a single founder. Eur J Hum Genet. 2013;21:102-8. doi:10.1038/ejhg. 2012.98.

4. Cruts M, Gijselinck I, Van Langenhove T, van der Zee J, Van Broeckhoven C. Current insights into the C9orf72 repeat expansion diseases of the FTLD/ALS spectrum. Trends Neurosci. 2013;36:450-9. doi:10.1016/j.tins.2013.04.010.

5. Zu T, Liu Y, Banez-Coronel M, Reid T, Pletnikova O, Lewis J, et al. RAN proteins and RNA foci from antisense transcripts in C9ORF72 ALS and frontotemporal dementia. Proc Natl Acad Sci U S A. 2013;110:E4968-77. doi:10.1073/pnas.1315438110.

6. Mori K, Weng SM, Arzberger T, May S, Rentzsch K, Kremmer E, et al. The C9orf72 GGGGCC repeat is translated into aggregating dipeptide-repeat proteins in FTLD/ALS. Science. 2013;339:1335-8. doi:10.1126/science. 1232927.

7. Ash PE, Bieniek KF, Gendron TF, Caulfield T, Lin WL, Dejesus-Hernandez M, et al. Unconventional translation of C9ORF72 GGGGCC expansion generates insoluble polypeptides specific to c9FTD/ALS. Neuron. 2013;77:639-46. doi:10.1016/j.neuron.2013.02.004.

8. Mori K, Arzberger T, Grasser FA, Gijselinck I, May S, Rentzsch K, et al. Bidirectional transcripts of the expanded C9orf72 hexanucleotide repeat are translated into aggregating dipeptide repeat proteins. Acta Neuropathol. 2013;126:881-93. doi:10.1007/s00401-013-1189-3.

9. Gendron TF, Bieniek KF, Zhang YJ, Jansen-West K, Ash PE, Caulfield T, et al. Antisense transcripts of the expanded C9ORF72 hexanucleotide repeat form nuclear RNA foci and undergo repeat-associated non-ATG translation in C9FTD/ALS. Acta Neuropathol. 2013;126:829-44. doi:10.1007/s00401-0131192-8.

10. Mizielinska S, Lashley T, Norona FE, Clayton EL, Ridler CE, Fratta P, et al. C9orf72 frontotemporal lobar degeneration is characterised by frequent neuronal sense and antisense RNA foci. Acta Neuropathol. 2013;126:845-57. doi:10.1007/s00401-013-1200-z.

11. Lagier-Tourenne C, Baughn M, Rigo F, Sun S, Liu P, Li HR, et al. Targeted degradation of sense and antisense C9orf72 RNA foci as therapy for ALS and frontotemporal degeneration. Proc Natl Acad Sci U S A. 2013;110: E4530-9. doi:10.1073/pnas.1318835110.

12. Donnelly CJ, Zhang P-W, Pham JT, Heusler AR, Mistry NA, Vidensky S, et al. RNA toxicity from the ALS/FTD C9ORF72 expansion is mitigated by antisense intervention. Neuron. 2013;80:415-28. doi:10.1016/j.neuron.2013. 10.015.

13. Lee $Y-B$, Chen $H-J$, Peres JN, Nishimura AL, Scotter E, Vance C, et al. Hexanucleotide repeats in ALS/FTD form length-dependent RNA foci, sequester RNA binding proteins and are neurotoxic. Cell Rep. 2013;5:117886. doi:10.1016/j.celrep.2013.10.049.

14. Almeida S, Gascon E, Tran H, Chou HJ, Gendron TF, Degroot S, et al. Modeling key pathological features of frontotemporal dementia with C9ORF72 repeat expansion in iPSC-derived human neurons. Acta Neuropathol. 2013;126:385-99. doi:10.1007/s00401-013-1149-y.

15. Sareen D, O'Rourke JG, Meera P, Muhammad AK, Grant S, Simpkinson M, et al. Targeting RNA foci in iPSC-derived motor neurons from ALS patients 
with a C9ORF72 repeat expansion. Sci Transl Med. 2013;5:208ra149. 10.1126/ scitranslmed.3007529.

16. Mori K, Lammich S, Mackenzie IR, Forne I, Zilow S, Kretzschmar H, et al. hnRNP A3 binds to GGGGCC repeats and is a constituent of p62-positive/ TDP43-negative inclusions in the hippocampus of patients with C9orf72 mutations. Acta Neuropathol. 2013;125:413-23. doi:10.1007/s00401-0131088-7.

17. Xu Z, Poidevin M, Li X, Li Y, Shu L, Nelson DL, et al. Expanded GGGGCC repeat RNA associated with amyotrophic lateral sclerosis and frontotemporal dementia causes neurodegeneration. Proc Natl Acad Sci U S A. 2013;110:7778-83. doi:10.1073/pnas.1219643110.

18. Cooper-Knock J, Walsh MJ, Higginbottom A, Robin Highley J, Dickman MJ, Edbauer D, et al. Sequestration of multiple RNA recognition motifcontaining proteins by C9orf72 repeat expansions. Brain. 2014;137:2040-51. doi:10.1093/brain/awu120.

19. Haeusler AR, Donnelly CJ, Periz G, Simko EAJ, Shaw PG, Kim M-S, et al. C9orf72 nucleotide repeat structures initiate molecular cascade of disease. Nature. 2014;507:195-200. doi:10.1038/nature13124.

20. Mizielinska S, Gronke S, Niccoli T, Ridler CE, Clayton EL, Devoy A, et al. C9orf72 repeat expansions cause neurodegeneration in Drosophila through arginine-rich proteins. Science. 2014;345:1192-4. doi:10.1126/science. 1256800.

21. May S, Hornburg D, Schludi MH, Arzberger T, Rentzsch K, Schwenk BM, et al. C9orf72 FTLD/ALS-associated Gly-Ala dipeptide repeat proteins cause neuronal toxicity and Unc119 sequestration. Acta Neuropathol. 2014;128: 485-503. doi:10.1007/s00401-014-1329-4.

22. Zhang YJ, Jansen-West $K$, Xu YF, Gendron TF, Bieniek KF, Lin WL, et al. Aggregation-prone c9FTD/ALS poly(GA) RAN-translated proteins cause neurotoxicity by inducing ER stress. Acta Neuropathol. 2014;128:505-24. doi:10.1007/s00401-014-1336-5.

23. Kwon I, Xiang S, Kato M, Wu L, Theodoropoulos P, Wang T, et al. Polydipeptides encoded by the C9orf72 repeats bind nucleoli, impede RNA biogenesis, and kill cells. Science. 2014;345:1139-45. doi:10.1126/science. 1254917.

24. Wen X, Tan W, Westergard T, Krishnamurthy K, Markandaiah SS, Shi Y, et al. Antisense proline-arginine RAN dipeptides linked to C9ORF72-ALS/FTD form toxic nuclear aggregates that initiate in vitro and in vivo neuronal death. Neuron. 2014;84:1213-25. doi:10.1016/j.neuron.2014.12.010.

25. Zhang K, Donnelly CJ, Haeusler AR, Grima JC, Machamer JB, Steinwald P, et al. The C9orf72 repeat expansion disrupts nucleocytoplasmic transport. Nature. 2015. doi:10.1038/nature14973.

26. Freibaum BD, Lu Y, Lopez-Gonzalez R, Kim NC, Almeida S, Lee KH, et al. GGGGCC repeat expansion in C9orf72 compromises nucleocytoplasmic transport. Nature. 2015;525:129-33. doi:10.1038/nature14974.

27. Jovicic A, Mertens J, Boeynaems S, Bogaert E, Chai N, Yamada SB, et al. Modifiers of C9orf72 dipeptide repeat toxicity connect nucleocytoplasmic transport defects to FTD/ALS. Nat Neurosci. 2015;18:1226-9. doi:10.1038/nn.4085.

28. Boeynaems S, Bogaert E, Michiels E, Gijselinck I, Sieben A, Jovicic A, et al. Drosophila screen connects nuclear transport genes to DPR pathology in C9ALS/FTD. Sci Rep. 2016;6:20877. doi:10.1038/srep20877.

29. Xiao S, MacNair L, McGoldrick P, McKeever PM, McLean JR, Zhang M, et al. Isoform specific antibodies reveal distinct subcellular localizations of C9orf72 in amyotrophic lateral sclerosis. Ann Neurol. 2015;78:568-83. doi:10.1002/ ana.24469.

30. Fratta P, Poulter M, Lashley T, Rohrer JD, Polke JM, Beck J, et al. Homozygosity for the C9orf72 GGGGCC repeat expansion in frontotemporal dementia. Acta Neuropathol. 2013;126:401-9.

31. Topisirovic I, Culjkovic B, Cohen N, Perez JM, Skrabanek L, Borden KL. The proline-rich homeodomain protein, $\mathrm{PRH}$, is a tissue-specific inhibitor of elF4E-dependent cyclin D1 mRNA transport and growth. EMBO J. 2003;22: 689-703. doi:10.1093/emboj/cdg069.

32. Topisirovic I, Guzman ML, McConnell MJ, Licht JD, Culjkovic B, Neering SJ, et al. Aberrant eukaryotic translation initiation factor $4 \mathrm{E}$-dependent mRNA transport impedes hematopoietic differentiation and contributes to leukemogenesis. Mol Cell Biol. 2003;23:8992-9002. doi:10.1128/MCB.23.24. 8992-9002.2003.

33. Davis BM, McCurrach ME, Taneja KL, Singer RH, Housman DE. Expansion of a CUG trinucleotide repeat in the $3^{\prime}$ untranslated region of myotonic dystrophy protein kinase transcripts results in nuclear retention of transcripts. Proc Natl Acad Sci U S A. 1997;94:7388-93. doi:10.1073/pnas.94. 14.7388 .
34. Taft RJ, Simons C, Nahkuri S, Oey H, Korbie DJ, Mercer TR, et al. Nuclearlocalized tiny RNAs are associated with transcription initiation and splice sites in metazoans. Nat Struct Mol Biol. 2010;17:1030-4. doi:10.1038/nsmb.1841.

35. Fukuchi $Y$, Nakajima H, Sugiyama D, Hirose I, Kitamura T, Tsuji K. Human placenta-derived cells have mesenchymal stem/progenitor cell potential. Stem Cells. 2004;22:649-58.

36. Wong JJ, Ritchie W, Ebner OA, Selbach M, Wong JW, Huang Y, et al. Orchestrated intron retention regulates normal granulocyte differentiation. Cell. 2013;154:583-95. doi:10.1016/j.cell.2013.06.052.

37. Shalgi R, Hurt JA, Lindquist $S$, Burge CB. Widespread inhibition of posttranscriptional splicing shapes the cellular transcriptome following heat shock. Cell Rep. 2014;7:1362-70. doi:10.1016/j.celrep.2014.04.044.

38. Clemson CM, Hutchinson JN, Sara SA, Ensminger AW, Fox AH, Chess A, et al. An architectural role for a nuclear noncoding RNA: NEAT1 RNA is essential for the structure of paraspeckles. Mol Cell. 2009;33:717-26. doi:10.1016/j. molcel.2009.01.026.

39. van Blitterswijk M, Gendron TF, Baker MC, DeJesus-Hernandez M, Finch NA, Brown PH, et al. Novel clinical associations with specific C9ORF72 transcripts in patients with repeat expansions in C9ORF72. Acta Neuropathol. 2015;130: 863-76. doi:10.1007/s00401-015-1480-6.

40. Hilleren PJ, Parker R. Cytoplasmic degradation of splice-defective pre-mRNAs and intermediates. Mol Cell. 2003;12:1453-65.

41. Tran H, Almeida S, Moore J, Gendron TF, Chalasani U, Lu Y, et al. Differential toxicity of nuclear RNA foci versus dipeptide repeat proteins in a Drosophila model of C9ORF72 FTD/ALS. Neuron. 2015;87:1207-14. doi:10.1016/j.neuron. 2015.09.015.

42. Braunschweig U, Barbosa-Morais NL, Pan Q, Nachman EN, Alipanahi B, Gonatopoulos-Pournatzis T, et al. Widespread intron retention in mammals functionally tunes transcriptomes. Genome Res. 2014;24:1774-86. doi:10. 1101/gr.177790.114.

43. Boutz PL, Bhutkar A, Sharp PA. Detained introns are a novel, widespread class of post-transcriptionally spliced introns. Genes Dev. 2015;29:63-80. doi:10.1101/gad.247361.114.

44. Hagerman P. Fragile X-associated tremor/ataxia syndrome (FXTAS): pathology and mechanisms. Acta Neuropathol. 2013;126:1-19. doi:10.1007/ s00401-013-1138-1.

45. Sathasivam K, Neueder A, Gipson TA, Landles C, Benjamin AC, Bondulich MK, et al. Aberrant splicing of HTT generates the pathogenic exon 1 protein in Huntington disease. Proc Natl Acad Sci U S A. 2013;110:2366-70. doi:10.1073/pnas.1221891110.

46. Raheem O, Olufemi SE, Bachinski LL, Vihola A, Sirito M, Holmlund-Hampf J, et al. Mutant (CCTG)n expansion causes abnormal expression of zinc finger protein 9 (ZNF9) in myotonic dystrophy type 2. Am J Pathol. 2010;177: 3025-36. doi:10.2353/ajpath.2010.100179.

47. Yap K, Makeyev EV. Regulation of gene expression in mammalian nervous system through alternative pre-mRNA splicing coupled with RNA quality control mechanisms. Mol Cell Neurosci. 2013;56:420-8. doi:10.1016/j.mcn. 2013.01.003.

48. Doma MK, Parker R. RNA quality control in eukaryotes. Cell. 2007;131:660-8. doi:10.1016/j.cell.2007.10.041.

49. Sommer P, Nehrbass U. Quality control of messenger ribonucleoprotein particles in the nucleus and at the pore. Curr Opin Cell Biol. 2005;17:294301. doi:10.1016/j.ceb.2005.04.007.

50. Rossi S, Serrano A, Gerbino V, Giorgi A, Di Francesco L, Nencini M, et al. Nuclear accumulation of mRNAs underlies G4C2 repeat-induced translational repression in a cellular model of C9orf72 ALS. J Cell Sci. 2015; 128:1787-99. doi:10.1242/jcs.165332.

51. Sun X, Li PP, Zhu S, Cohen R, Marque LO, Ross CA, et al. Nuclear retention of full-length HTT RNA is mediated by splicing factors MBNL1 and U2AF65. Sci Rep. 2015;5:12521. doi:10.1038/srep12521.

52. Rougemaille M, Villa T, Gudipati RK, Libri D. mRNA journey to the cytoplasm: attire required. Biol Cell. 2008;100:327-42. doi:10.1042/BC20070143.

53. Houseley J, Tollervey D. The many pathways of RNA degradation. Cell. 2009; 136:763-76. doi:10.1016/j.cell.2009.01.019.

54. Lemieux C, Marguerat S, Lafontaine J, Barbezier N, Bahler J, Bachand F. A Pre-mRNA degradation pathway that selectively targets intron-containing genes requires the nuclear poly(A)-binding protein. Mol Cell. 2011;44:10819. doi:10.1016/j.molcel.2011.06.035.

55. Bergeron D, Pal G, Beaulieu YB, Chabot B, Bachand F. Regulated intron retention and nuclear pre-mRNA decay contribute to PABPN1 autoregulation. Mol Cell Biol. 2015. doi:10.1128/MCB.00070-15. 
56. Bresson SM, Conrad NK. The human nuclear poly(a)-binding protein promotes RNA hyperadenylation and decay. PLoS Genet. 2013;9:e1003893. doi:10.1371/journal.pgen.1003893.

57. Fratta P, Mizielinska S, Nicoll AJ, Zloh M, Fisher EM, Parkinson G, et al. C9orf72 hexanucleotide repeat associated with amyotrophic lateral sclerosis and frontotemporal dementia forms RNA G-quadruplexes. Sci Rep. 2012;2: 1016. doi:10.1038/srep01016.

58. Reddy K, Zamiri B, Stanley SY, Macgregor RB, Pearson CE. The diseaseassociated r(GGGGCC)n repeat from the C9orf72 gene forms tract lengthdependent uni- and multi-molecular RNA G-quadruplex structures. J Biol Chem. 2013:288:9860-6. doi:10.1074/jbc.C113.452532.

59. Su Z, Zhang Y, Gendron TF, Bauer PO, Chew J, Yang WY, et al. Discovery of a biomarker and lead small molecules to target r(GGGGCC)-associated defects in C9FTD/ALS. Neuron. 2014;83:1043-50. doi:10.1016/j.neuron.2014. 07.041.

60. Skourti-Stathaki K, Kamieniarz-Gdula K, Proudfoot NJ. R-loops induce repressive chromatin marks over mammalian gene terminators. Nature. 2014;516:436-9. doi:10.1038/nature13787.

Submit your next manuscript to BioMed Central and we will help you at every step:

- We accept pre-submission inquiries

- Our selector tool helps you to find the most relevant journal

- We provide round the clock customer support

- Convenient online submission

- Thorough peer review

- Inclusion in PubMed and all major indexing services

- Maximum visibility for your research

Submit your manuscript at www.biomedcentral.com/submit
Biomed Central 